\title{
Chidamide and 5-flurouracil show a synergistic antitumor effect on human colon cancer xenografts in nude mice
}

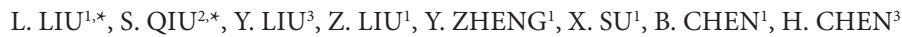 \\ ${ }^{1}$ Department of Oncology, Zhong-Da Hospital, School of Medicine, Southeast University, 87 Ding Jia Qiao, Nanjing210009, PR China; ${ }^{2}$ Depart- \\ ment of Internal Medicine, Nanjing Municipal Tumor Hospital, 1-1 Zhong Fu Road, Nanjing210002, PR China; ${ }^{3}$ Department of Digestology, \\ Zhong-Da Hospital, School of Medicine, Southeast University, 87 Ding Jia Qiao, Nanjing210009, R China
}

*Correspondence: wenyu811@126.com,qiushaomin60@sohu.com

Received April 22, 2015 / Accepted September 7, 2015

\begin{abstract}
Chidamide is a novel histone deacetylase (HDAC) inhibitor that increases the acetylation of histone $\mathrm{H} 3$ by inhibiting the activity of HDAC1 and HDAC2. We previously found that treatment of human colon cancer cells with chidamide led to cell apoptosis and cell cycle arrest at G0/1 phase in vitro. The present study extended the observations in vivo and explored the underlying molecular mechanisms. In nude mice bearing human colon cancer LoVo cell xenografts, chidamide alone or in combination with 5-flurouracil (5-Fu) reduced the expression of HDAC1 and HDAC2, accompanied with increased acetylation of histone H3. Chidamide alone inhibited the tumor growth and induce cell apoptosis in tumor-bearing mice. Combined treatment of chidamide with 5-Fu enhanced the anti-tumor activity of 5-Fu. Western blotting analysis showed that chidamide alone or in combination with 5-Fu upregulated the expressions of cleaved Caspase-3 and cleaved poly-ADP (adenosine diphosphate)-ribose polymerase (PARP). In addition, chidamide alone or in combination with 5-Fu increased the p53, phosphorylated-p53 (p-p53), p21 and $\gamma \mathrm{H} 2 \mathrm{AX}$ levels, but suppressed cyclin dependent kinase 4 (CDK4) expression in tumor cells. Chidamide alone or in combination with 5-Fu down regulated the expressions of p-AKT, p-mammalian target of rapamycin (mTOR), p-p70S6K, p-Raf, and p44/42 mitogen activated protein kinase (Erk1/2), indicating the blockage of these signaling pathways. The results demonstrated that chidamide alone or in combination with 5-Fu exerted anti-tumor activity in nude mice bearing human colon cancer LoVo cell xenografts, and several signaling pathways might be involved in the chidamide-induced tumor growth inhibition and tumor cell apoptosis.
\end{abstract}

Key words: HDAC inhibitor, chidamide, colon cancer, 5-flurouracil

Colorectal cancer is one of the most frequently diagnosed malignant neoplasms in clinic and increasing cases are reported in young people [1]. Thus, prevention and treatment of colorectal cancer has become a serious public health concern. Surgical removal is the most effective and first choice for the treatment of colorectal cancer, however, $30-40 \%$ colorectal cancer patients die from recurrence and metastasis. Moreover, approximately $20 \%$ colorectal cancers are diagnosed at late stages, when surgery is not suitable [2]. Cytotoxic chemotherapy is the current strategy for the management of these cancer patients with relapse, metastasis and later stages [3]. 5 -flurouracil $(5-\mathrm{Fu})$ is used for the treatment of advanced colorectal cancer since 1957, and is still the basic drug for systematic chemotherapy of colorectal cancer. About 80-90\% colorectal cancers are adenocarcinoma with slow proliferation and resistant to conventional chemotherapies due to the primary and secondary multidrug resistance. Therefore, it is appealing to develop novel therapeutic modalities for the treatment of patients with colorectal cancers especially those with relapse, metastasis and later stages.

Cancer is a disease resulting from a combined accumulation of genetic and epigenetic alterations [4] Recently, with the increased understanding of the critical roles of epigenetic modification of chromatin in the tumorigenesis and anticancer drug resistance, targeting epigenetic modifiers of chromatin is a new trend for the development of anticancer drugs [5]. Histone deacetylase inhibitor (HDACi) is a group of the most extensively and intensively studied and developed agents that target epigenetic modifiers, and has become a potential general strategy to reverse the epigenetic alterations of cancer cells [6]. HDACi affects gene transcription by changing the conformation of chromosomes. Apparently HDACi 
is distinct from conventional cytotoxic hemotherapies and molecular targeted therapy of tyrosine kinase inhibitors. Moreover, HDACi had shown synergistic effects with other therapeutic agents [7]. Therefore, development of HDACi is a very promising alternative strategy to improve the effects of other therapeutic modules and thus the life quality of cancer patients.

Deregulation of histone acetylation was reported to play key roles during the initiation, progression and maintenance of colorectal cancer [8]. Accordingly, several HDACi, including vorinostat, romidepsin, MS-275 and TSA have been demonstrated to inhibit the growth of lots of colorectal cancer cells in vitro [9]. These facts suggest that HDACi is a promising potential choice for the targeted therapy of colorectal cancers. To date, two HDACi (vorinostat and romidepsin) have been approved by US Food and Drug Administration (FDA) for the clinical treatment of skin T cell lymphoma [10]. Currently, numerous HDACi are under pre-clinical trials and more than $10 \mathrm{HDACi}$ are in clinical trials [11]. Furthermore, the combination therapy of HDACi with other anticancer therapeutic modules is also under extensive investigation both in academic and pharmaceutical companies [12].

Chidamide is a novel HDACi with improved chemical structure and marked anti-tumor activity [13-14]. Our previous study reported that chidamide induced cell apoptosis and cell cycle arrest at G0/1 phase of human colon cancer cells cultured in vitro [15]. However, the in vivo antitumor effects of chidamide on human colon cancer remain unexplored. The present study investigated the therapeutic effects of chidamide in mouse tumor xenografts combined with 5 -Fu and explored the underlying molecular mechanisms by which chidamide combined with 5-Fu effect the human colon cancer.

\section{Materials and methods}

Chemicals. Chidamide was provided by Shenzhen Chipscreen Biosciences (Shenzhen, China). 5-flurouracil (5-Fu) was purchased from Jingyao Amino Acids (Tianjin, China). Primary antibodies including anti-acetyle- $\mathrm{H} 3$, anti-cleaved Caspase-3, anti-PARP, anti-CKD4, anti-p-p38, anti-JNK1, anti-p-JNK1, anti-p-Akt (Ser473), anti-mTOR, anti-pp70S6K, anti-p70S6K, anti-p-Erk, and anti-Erk antibodies were purchased from Cell Signaling Technology (Boston, Massachusetts, USA). Primary antibodies, including anti-p53, anti-p-p53-S15, anti-p21, anti- $\gamma \mathrm{H} 2 \mathrm{AX}$, anti-Bax, anti-Bak, anti-Bcl-2, anti-MDM2, anti-p38, anti-Raf-1, anti-p-Raf, and anti-GAPDH antibodies were obtained from SantaCruz Biotechnology (Dallas, Texas, USA). Anti-HDAC1 and anti-HDAC2 antibodies were bought from Epigentek (Farmingdale, NewYork, USA).

Cell line and mouse tumor xenograft models. The present animal experiment was approved by the Animal Care and Use Committee of Southeast University, Nanjing, China. Human colorectal adenocarcinoma anaplastic LoVo cell line was bought from the cell bank of Shanghai Institute of Cell Biology, Chinese Academy of Sciences (Shanghai, China). Exponentially growing LoVo cells were collected and injected subcutaneously into the right axilla of 5 nude mice. Three days after implantation, palpable tumors were detected in 3 mice. Three weeks later, one mouse with tumor size of $1 \mathrm{~cm} \times 0.8 \mathrm{~cm} \times 0.8 \mathrm{~cm}$ was sacrificed. The tumor mass was cut into slices of $1-2 \mathrm{~mm}^{3}$ and transplanted subcutaneously into the right axilla of 4 nude mice and propagated the same way for 3 passages. During the $4^{\text {th }}$ transplantation, tumor xenografts were implanted into 50 nude mice. Ten days later, 45 mice developed tumors.

Drug treatment. Thirty-two nude mice of the same age were randomly divided into 4 groups with 8 animals for each group. In the first group (chidamide group), mice were treated with chidamide $5 \mathrm{mg} / \mathrm{kg} / \mathrm{d}$ via intragastric administration. The second group animal (5-Fu group) was treated with 5-Fu 25 $\mathrm{mg} / \mathrm{kg} / \mathrm{d}$ through intraperitoneal injection. In the third group (Chidamide +5 -Fu group), mice were treated with chidamide $5 \mathrm{mg} / \mathrm{kg} / \mathrm{d}$ (intragastric administration) and 5-Fu $25 \mathrm{mg} /$ $\mathrm{kg} / \mathrm{d}$ (intraperitoneal injection). In the fourth group (negative control group), mice were treated with vehicle by intragastric administration. The treatment of the mice was carried out on daily bases for 21 consecutive days.

Observation of tumor xenografts. The maximum length (a) and width (b) of each tumor were measured at the first day of drug administration and followed by measurement every 3 days. Daily observations of spirit, diet, activities, and defecation of mice were recorded. Mice were weighted every day and drugs were administrated every day for 21 consecutive days. In the $22^{\text {nd }}$ day, the mice weight was recorded and the maximum length (a) and width (b) were measured with a vernier caliper. The following formula were used to calculate tumor volume and tumor volume suppression rate: tumor volume $(V)=1 / 2 \mathrm{ab}^{2}$; tumor volume suppression rate $=(1-$ average tumor volume of experiment group / average tumor volume of control group) $\times 100 \%$. The mice were sacrificed and the tumors were removed and weighted. The tumor suppression rates were calculated according to the following formula: tumor-suppression rate $=(1-$ average tumor weight of experiment group / average tumor weight of control group) $\times 100$ $\%$. Coefficient of drug interaction (CDI) was used to evaluate the interaction of the two drugs and computed according to the following formula: $\mathrm{CDI}=\mathrm{AB} /(\mathrm{A} \times \mathrm{B})$. $\mathrm{AB}$ is the tumor weight (or volume) ratio of combinatory group to the control group. A or B is the tumor weight (or volume) ratio of single drug group to the control group. $\mathrm{CDI}<1$ indicates synergistic effect, $\mathrm{CDI}=1$ indicates additive effects and CDI $>1$ indicates antagonistic effects of the two drugs.

Histological and ultra-thin slices observation. Tumors of xenografts from each group were stained with conventional $\mathrm{H}-\mathrm{E}$ staining. Changes of cellular morphology and tissue structures were observed under light microscope and recorded by photograph. Ultra-thin slices were double stained with uranium and lead, and observed under JEM - 1011 transmission electron microscope (TEM). 
Western blotting. Tumor tissues were grinded on dry ice and then lysed on ice in cell lysis buffer. Protein quantification was performed with the BCA protein assay (Pierce, Rockford, IL, USA). Equal amounts of protein from each sample were denatured in SDS sample buffer and separated on 10\% SDS polyacrylamide gel. Proteins were transferred to nitrocellulose membranes. Membranes were blocked, and then probed with primary antibody ( $1: 1000$ dilution) at $4^{\circ} \mathrm{C}$ for overnight. After washing, membranes were incubated with secondary antibody at room temperature for $2 \mathrm{~h}$. Immunobands were visualized using enhanced chemiluminescence (ECL) kit (Pierce, Rockford, IL, USA). Relative protein levels were analyzed with Gene Tools software.

Statistical analysis. Statistical analysis was conducted using SPSS 16.0 (SPSS Inc., Chicago, IL, USA). All data are presented as mean $\pm S D$. Significant differences were determined using analysis of variance. $P$-values were two-sided, and $p$-values $<$ 0.05 were considered statistically significant.

\section{Results}

Chidamide significantly enhances the antitumor effects of 5-Fu in human colon cancer nude mouse xenografts. We determined the protein levels of HDAC1, HDAC2 and acetylated total $\mathrm{H} 3$ in nude mice bearing human colon cancer LoVo cell xenografts after treatment with chidamide alone or chidamide in combination with 5-Fu by immunoblots. Compared with vehicle control and 5-Fu, chidamide apparently decreased the protein levels of HDAC1 and HDAC2, and accordingly increased acetylated $\mathrm{H} 3$ in the presence or absence of 5-Fu (Fig. 1A).

All the mice survived till the end of the experiments. No mouse had diarrhea in the vehicle control group. Slight diarrhea was observed in chidamide group whereas, high frequent diarrhea was found in the groups of 5-Fu and chidamide plus 5 -Fu. In comparison to vehicle control group, chidamide did not change the weight of mice apparently, while treatment with either 5-Fu or 5-Fu plus chidamide led to significant loss of body weight $(p<0.01)$ (Fig. 1B).

Compared with vehicle control, chidamide or 5-Fu alone, chidamide plus $5-\mathrm{Fu}$ apparently inhibited tumor growth. Statistically significant differences were found among the 4 groups 10 days after drug administration (Fig. 1C). By the end of drug administration, the tumor volume suppression rates of 5-Fu alone, chidamide alone, and chidamide plus 5 -Fu were $48.60 \%, 34.71 \%$ and $73.25 \%$, respectively. The tumor volume suppression rate of chidamide plus 5 -Fu was significantly higher than those of $5-\mathrm{Fu}$ and chidamide $(p=$ $0.000, p=0.000$ ) (Fig. 1C). Tumor weight suppression rates of 5 - Fu alone, chidamide alone, and chidamide plus $5-\mathrm{Fu}$ were $43.52 \%, 31.36 \%$ and $72.30 \%$, respectively. Similar to the tumor volume suppression rate, the tumor weight suppression rate of combined therapy was significantly higher than that of single drug administration $(p<0.01)$ (Fig. 1D-F). The CDI values of tumor size and tumor weight were lower than
1, suggesting chidamide and 5-flurouracil show a synergistic antitumor effect.

$\mathrm{H}$-E staining showed that the tumor tissue structure of negative controls was normal with reduced interfibrillar substance, relative large tumor cell size, a multi-shaped cell morphology, large and deep-stained nucleus, and relative less cytoplasm; there was large area of necrotic tumor tissue with sporadic distribution of apoptotic cells in 5-Fu group. In chidamide group, there was only slight change in the tissue structure with discernable tumor cell necrosis and apoptosis. In contrast, in chidamide plus 5-Fu group, there was extended necrotic area with apparent cell apoptosis (Fig. 1G). These results together demonstrated that chidamide significantly enhanced the antitumor effects of 5 -Fu in nude mouse bearing human colon cancer xenografts.

Chidamide in combination with 5-Fu results in enhanced cell apoptosis in nude mouse bearing human colon cancer xenografts. To investigate whether chidamide sensitizes colon cancer cells to 5-Fu via enhancing 5-Fuinduced apoptosis, we determined cell apoptosis of tumor xenografts treated with chidamide alone or in combination with 5-Fu by transmission electron microscope, the activation of apoptosis-related proteins by western blots. Transmission electron microscope showed that 5-Fu treatment led to the reduction of cell density, disruption of cytoplasm membrane, release of intracellular constituents, dissolution of cytoplasm and chromatin, and disruption of nucleolus and nuclear membrane. Chidamide treated mice showed an increase of clustered heterochromatin with some cystoid expansion and the space between cytoplasm and nucleus membranes, swelling mitochondrion with partially or completely disappeared ridges, expansion of endoplasmic reticulum with cystoid-like structure, and a change of the micro-structural characteristics of degenerated cells in tumor cells. Moreover, some cells shrinked as demonstrated by increased electron density of cytoplasm, cytoplasm bubbles and apparent apoptotic bodies but intact cytoplasm membrane, suggesting cell apoptosis. Similar changes in the micro-structural characteristics and apoptotic cells were observed in chidamide plus 5-Fu group (Fig. 2A). In consistent with these morphologic observations, there were increased cleavage of both Caspase-3 and PARP in chidamide or chidamide plus 5-Fu groups compared with those of negative control and 5-Fu groups (Fig. 2B). These results indicated that chidamide alone induced cell apoptosis, and the combination of chidamide and 5-Fu treatment enhanced cell apoptosis.

Chidamide treatment results in phosphorylation of $\mathrm{H} 2 \mathrm{AX}$ and $\mathrm{p} 53$ in nude mouse bearing human colon cancer xenografts. We previously found that chidamide led to cell cycle arrest at G0/G1 phase with an increase of p21 while a decrease of CDK4 in LoVo and HT-29 cells ${ }^{[14]}$. To assess the mechanisms by which chidamide induces cell apoptosis and enhances the anti-tumor effects of 5-Fu in vivo, the protein levels of $\mathrm{p} 21, \mathrm{CDK} 4, \mathrm{Bax}, \mathrm{Bak}$, and Bcl-2 were examined in the xenografts. Compared with those of negative control and 
A

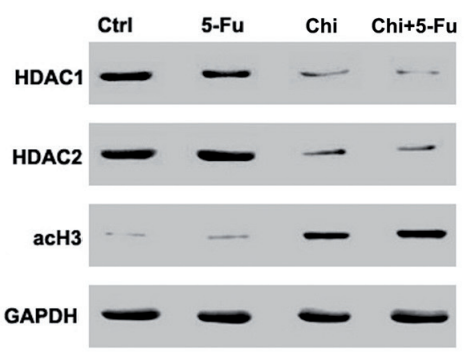

C

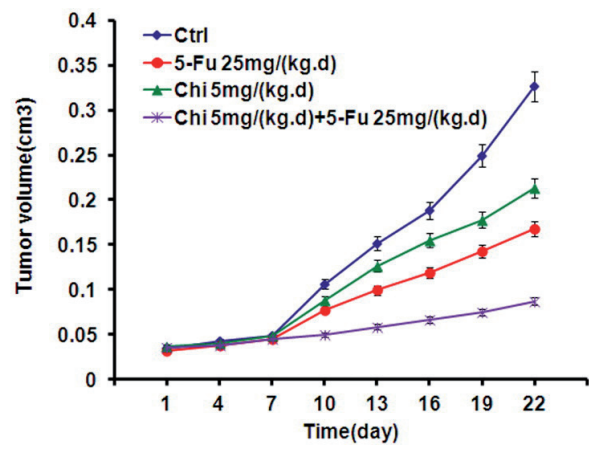

$\mathbf{E}$

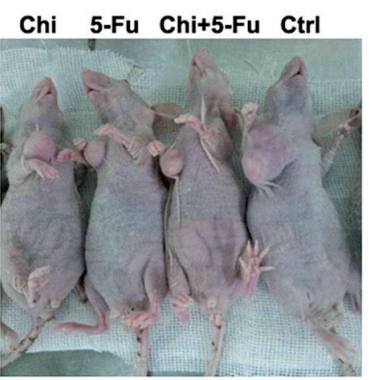

B

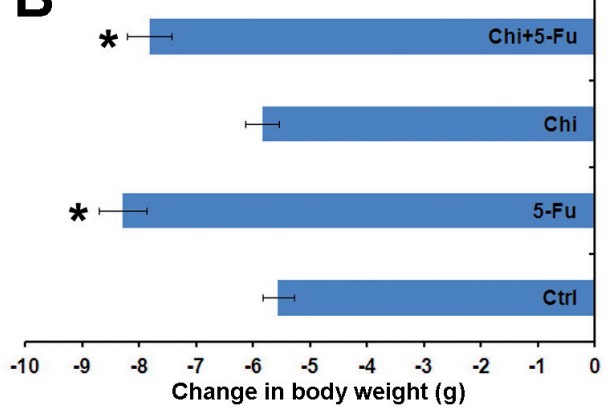

D

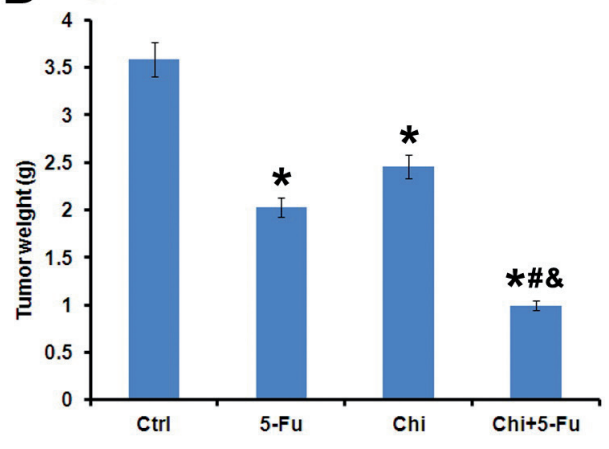

E Chi 5-Fu Chi+5-Fu Ctrl

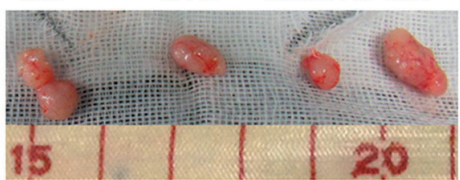

G

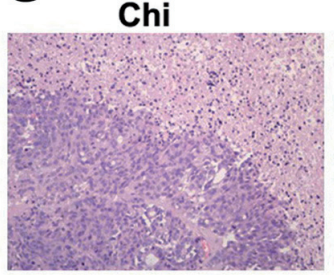

5-Fu

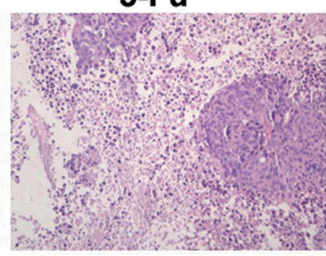

Chi+5-Fu

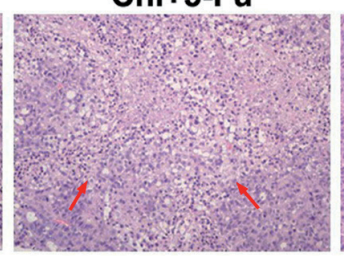

\section{Ctrl}

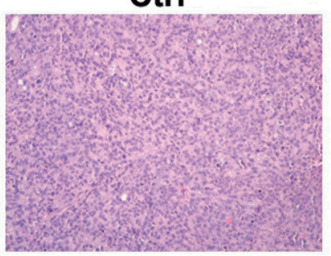

Figure 1. Chidamide synergies with 5-Fu in suppressing the growth of human colon cancer nude mouse xenografts. (A) Human colon cancer nude mouse xenografts were propagated subcutaneously in nude mice and were treated as the indicated drugs. Tumors were harvested 22 days post treatment. Total proteins were extracted for immunoblotting of HDAC1, HDAC2, Acyt total H3 and GAPDH. (B) Human colon cancer nude mouse xenografts were propagated subcutaneously in nude mice and were treated as indicated. Mice were weighted every day. The average mouse weight in the $22^{\text {nd }}$ day was shown ( $p<0.05$ vs negative control group). (C) Mouse tumor xenografts were treated as indicated. In the $22^{\text {nd }}$ day, the maximum length (a), width (b) and height (c) of tumors were measured with a vernier caliper. Tumor volume was calculated according to the following formula: tumor volume $(\mathrm{V})=$ $1 / 2 \mathrm{ab}^{2}$. (D) Mouse tumor xenografts were treated as indicated. In the $22^{\text {nd }}$ day, the mice were sacrificed and the subcutaneous transplant tumors were removed and weighted $\left({ }^{*} p<0.05\right.$ vs negative control group; ${ }^{*} p<0.05$ vs 5 -Fu group; and ${ }^{\circledR} p<0.05$ vs Chidamide group). (E) Representative mice following treatment with the indicated drugs were shown. (F) Representative tumor xenografts from mice following treatment with the indicated drugs were shown. (G) H-E staining of representative tumor xenografts from mice following treatment with the indicated drugs was shown. Chidamide plus 5-Fu induced extended necrosis with apparent cell apoptosis (red arrow head)(HE stain; magnification, $\times 200)$. 


\section{A}
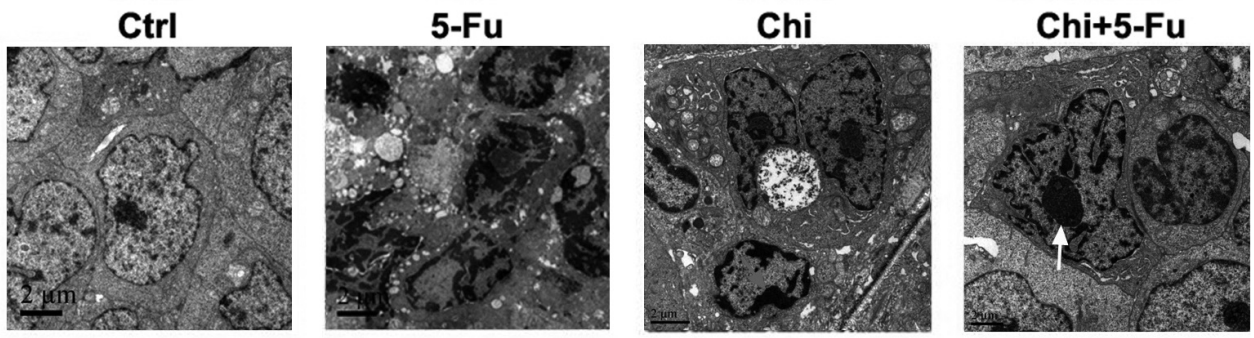

B

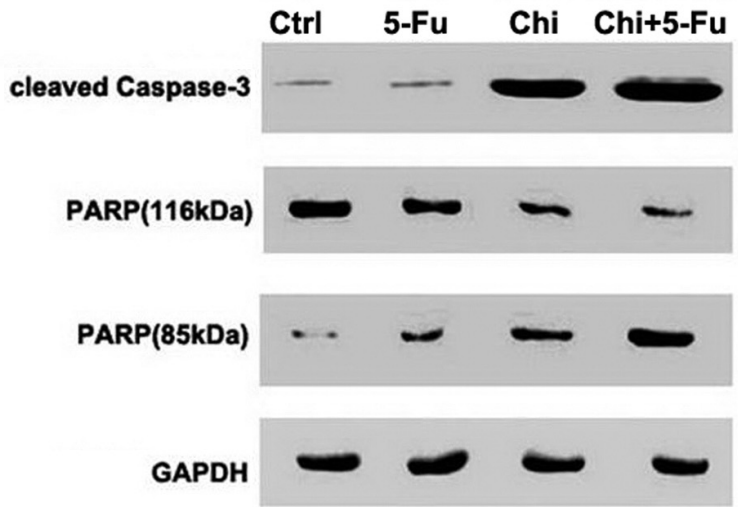

Figure 2. Chidamide combined with 5-Fu results in enhanced cell apoptosis in LoVo cell xenografts.. (A) Apoptotic cells of tumor xenografts treated as indicated were determined by transmission electronic microscope. Chidamide and 5-Fu induced apoptosis, as evidenced by chromatin margination (red arrow head) and nuclear condensation (white arrow head). (B) Human colon cancer nude mouse xenografts were harvested 22 days post treatment. Total proteins were extracted for immunoblotting of cleaved Caspase 3, cleavage and total PARP, and GAPDH.

5-Fu groups, chidamide apparently increased p21, Bax, and Bak while decreased CDK4 and Bcl-2. As p21, Bax, Bak, and $\mathrm{Bcl}-2$ is the direct downstream target of p53. Moreover, chidamide inhibited the expression of MDM2. Our data suggest that chidamide may activate the p53 signaling pathway. Indeed, chidamide induced dramatic phosphorylation of p53 at S15 in comparison to vehicle. 5-Fu is a well-known DNA damage agent that induces phosphorylation of p53 at S15 in various types of cells. Accordingly, 5-Fu induced phosphorylation of $\mathrm{H} 2 \mathrm{AX}$ and $\mathrm{p} 53$ in the colon cancer LoVo cell xenografts in nude mice. Interestingly, it was found that chidamide alone induced apparent phosphorylation $\mathrm{H} 2 \mathrm{AX}$, a marker of DNA strand breaks. Moreover, enhanced phosphorylation of $\mathrm{H} 2 \mathrm{AX}$ and p53, and up-regulation of the protein levels of p21, Bax, Bak, p53 and MDM2, and down-regulation of CDK4 and Bcl-2 were observed in human colon cancer LoVo cell xenografts treated with both chidamide and 5-Fu (Fig. 3). These results suggest that one of the mechanisms of chidamide-induced tumor cell apoptosis may be through changing chromatin structure and hence DNA damage.

Chidamide suppresses the AKT-mTOR and Raf-MEKERK signaling pathways in nude mouse bearing human colon cancer xenografts. To further investigate the mechanisms by which chidamide inhibits cell cycle progression, we determined the activation of AKT-mTOR and MAPK pathways in the colon cancer LoVo cell xenografts treated with chidamide, 5-Fu alone or in combination by immunoblots. Compared with those of negative control and 5-Fu, chidamide apparently decreased the phosphorylation of AKT (Ser473), mTOR and p70S6K, indicating that chidamide suppresses AKT-mTOR signaling pathways. Moreover, chidamide reduced the phosphorylation of Raf and Erk1/2, but neither JNK nor p38 pathways were apparently altered by Chidamide, indicating suppression of Raf-MEK-ERK signaling by chidamide. However, 5-Fu alone did not alter the phosphorylation status of AKT, mTOR, p70S6K, Raf and Erk1/2. Furthermore, similar signaling pathway suppression pattern was observed in combination treatment group (Fig. 4).

\section{Discussion}

In this study, using nude mouse bearing human colon cancer xenografts, we found that chidamide inhibited HDAC1 and $\mathrm{HDAC} 2$, and hence increased the acetylation of histone $\mathrm{H} 3$. Chidamide inhibited the tumor growth of LoVo xenografts in nude mice, accompanied with increased cleavage of Caspase- 3 and PARP and down-regulation of the phosphorylation of AKT (Ser473), mTOR, p70S6K, Raf and Erk1/2. In addition, 
it was we found that chidamide induced the phosphorylation of $\mathrm{H} 2 \mathrm{AX}$ and $\mathrm{p} 53$ while and increased the levels of p21. Furthermore, chidamide significantly enhanced the anti-tumor effects of 5-Fu in nude mouse bearing human colon cancer xenografts. These results suggest the alteration of chromatin remodeling by chidamide may lead to DNA damage response and provide the basis for further development of chidamide as a novel HDACi for cancer treatment as single agent or in combination with other therapeutic modules.

Chidamide is a HDACi of benzamides with improved chemical structure and has been shown to inhibit the growth

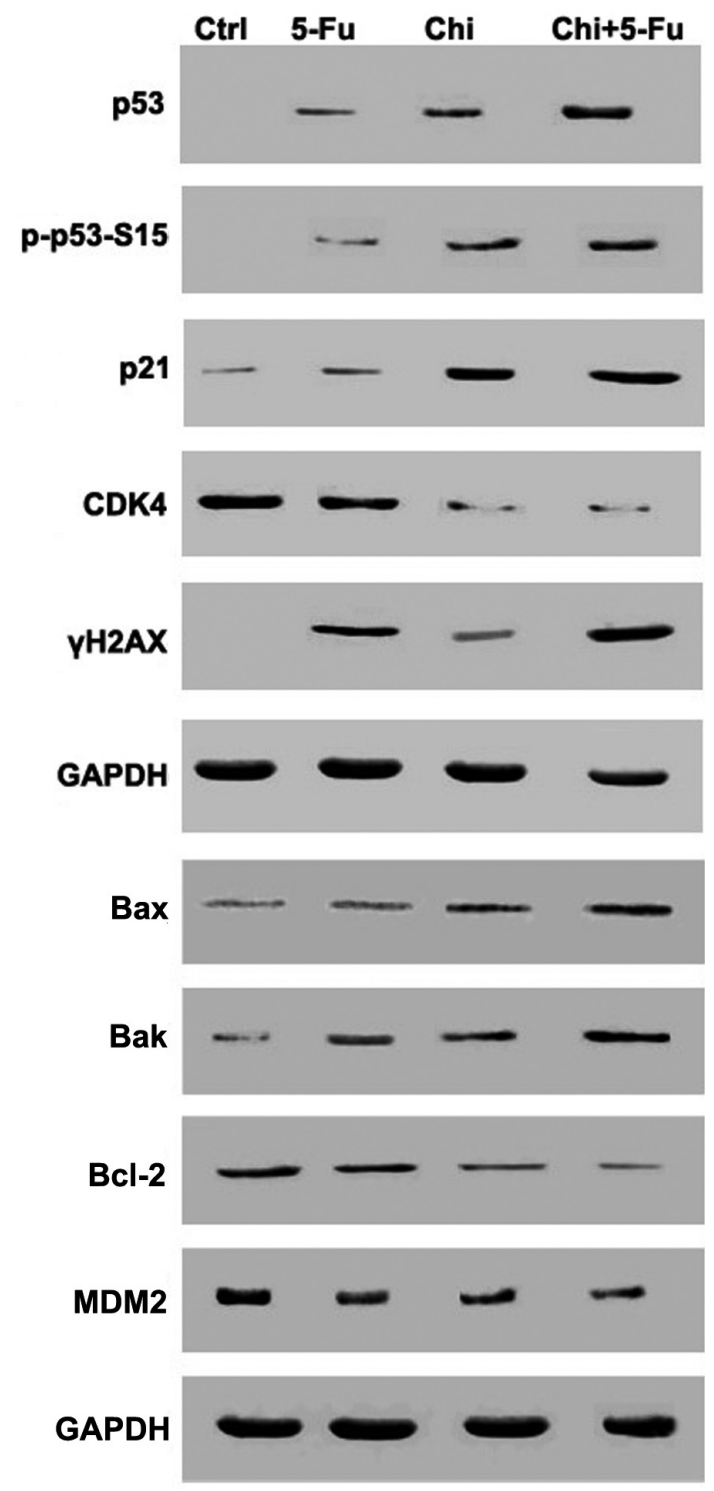

Figure 3. Chidamide treatment resulted in DNA damage response in colon cancer LoVo cell nude mouse xenografts. Human colon cancer nude mouse xenografts were propagated subcutaneously in nude mice and were treated as indicated. Tumors were harvested 22 days post treatment. Total proteins were extracted for immunoblotting of total p53, p-p53-S15, p21, CDK4, $\gamma \mathrm{H} 2 \mathrm{AX}, \mathrm{Bax}, \mathrm{Bak}, \mathrm{Bcl}-2, \mathrm{MDM} 2$ and GAPDH.

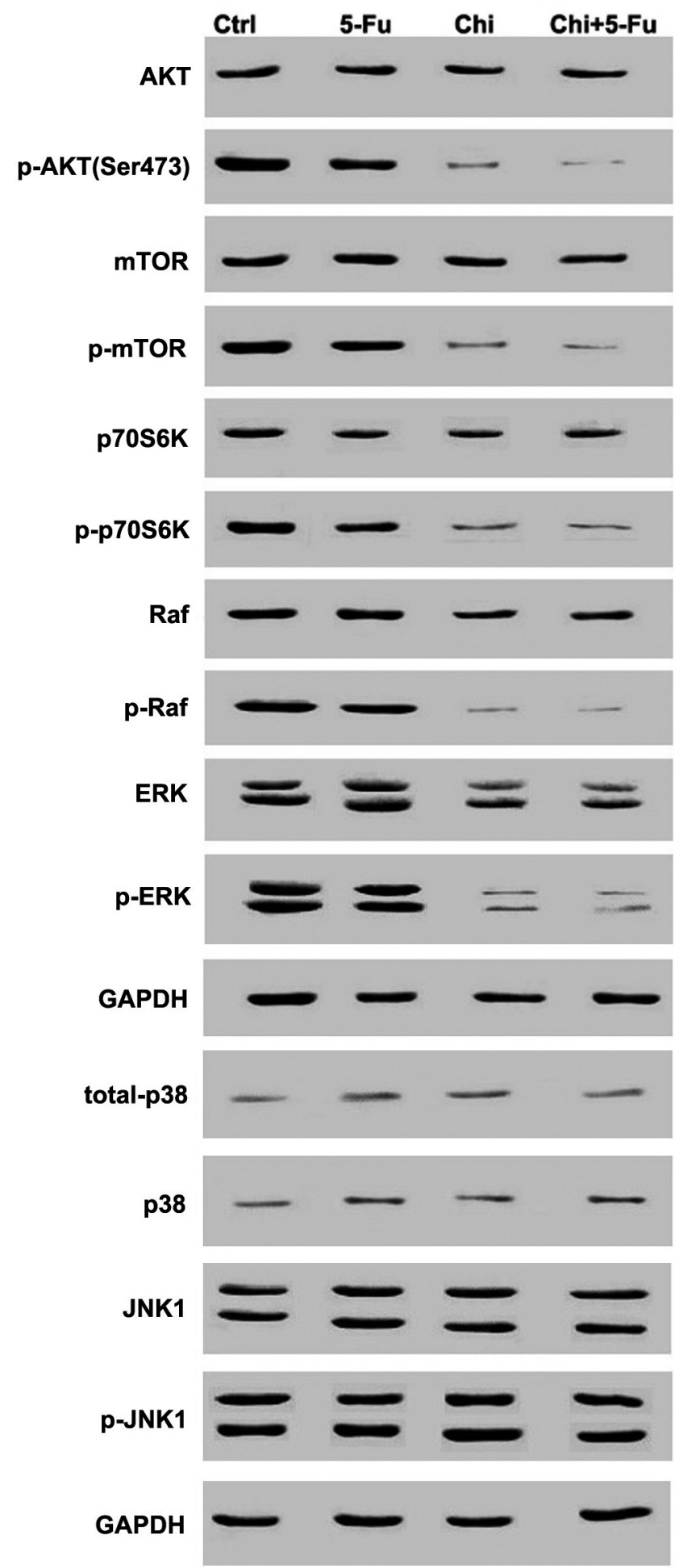

Figure 4. Chidamide treatment surpressed AKT-mTOR and Raf-MEKERK signaling pathways in LoVo cell xenografts. Human colon cancer nude mouse xenografts were propagated subcutaneously in nude mice and were treated as indicated. Tumors were harvested 22 days post treatment. Total proteins were extracted for immunoblotting of p-AKT, AKT, p-mTOR, mTOR, p-p70S6K, p70S6K, p-Raf, Raf, p-Erk1/2, Erk1/2,p-p38, total-p38, p-JNK1, JNK1 and GAPDH. 
of lymphoma, leukemia, liver carcinoma, pancreatic cancers and non-small cell lung cancers efficiently [13-20]. Chidamide may prevent cell proliferation, whereas promote cell apoptosis and dedifferentiation by altering gene expression through specifically inhibiting some subtypes of HDAC.

Our previous study demonstrated that chidamide induced cell apoptosis and cell cycle arrest at G0/1 phase of human colon cancer LoVo and HT-29 cells cultured in vitro [15]. In addition, chidamide promoted the cleavage of Caspase- 3 and up-regulation of $\mathrm{p} 21$, whereas, decreasesd the expression of CDK4 [15]. Moreover, chidamide was found to inhibit the activity of the AKT/mTOR and Raf-MEK-ERK signaling pathways [15]. In the present study, we extended these observations in mouse tumor xenografts and found that chidamide sensitized colorectal cancers to 5-Fu. Most importantly, it was found that Chidamide induced DNA strand breaks in vivo.

Similar to the previous in vitro study [15], chidamide decreased the levels of HDAC1 and HDAC2, accompanied with increased acetyl-H3, in colon cancer LoVo cell xenografts of nude mice. Moreover, chidamide alone apparently inhibited the growth of colon cancer cells. Enhanced anti-tumor activity and increased cell apoptosis was observed by combined administration of chidamide with 5 -Fu. It is possible that chidamide plus $5-\mathrm{Fu}$ therapy may promote tumor growth inhibition and tumor cell apoptosis induced by single drug treatment by increasing the cleavage of Caspase-3 and PARP.

Noticeably, the present study revealed that chidamide induced apparent DNA strand breaks as indicated by the increased level of $\gamma \mathrm{H} 2 \mathrm{AX}$, a marker of DNA double strand breaks. DNA damage leads to activation of ATM/Chk2 and ATR/Chk1 DNA damage checkpoints, both of which phosphorylate and hence activate the central tumor suppressor p53 [21-24]. In consistent with the phosphorylation of H2AX, treatment of chidamide led to phosphorylation of $\mathrm{p} 53$ at ser15, accompanied with increased protein level of p21, Bax, and $\mathrm{Bak}$, and decreased protein level of $\mathrm{Bcl}-2$, the direct targets of p53. Furthermore, expression of p53-related gene MDM2 was also suppressed. The main physiologic function of ATM/ Chk2 and ATR/Chk1 DNA damage checkpoints is to arrest cell cycle progression, promote DNA damage repair and cell apoptosis [22-24]. In consistent, our previous in vitro and the present in vivo studies showed that chidamide induced cell cycle arrest at G0/G1 phase and cell apoptosis. In addition, chidamide suppressed the activity of the AKT/mTOR and Raf-MEK-ERK pathways, which is in agreement with the observation that ATM/Chk2 and ATR/Chk1 DNA damage checkpoints suppress the mTOR and MAPK pathways [25-26]. Our study found neither JNK nor p38 pathways were apparently altered by Chidamide. These data suggest that one of the mechanisms by which chidamide induces cell cycle arrest and apoptosis might be through induction of DNA damages. However, the underlying molecular mechanism still needs to be further clarified.
According to the tumor volume suppression rate and the tumor weight suppression rate, the anti-tumor effect of Chidamide seems to be weaker than that of 5-Fu. However, the effect of Chidamide is stronger than that of $5-\mathrm{Fu}$, regarding to the activation of caspase and suppression of AKT-mTOR and Raf-MEK-ERK signaling. Such discrepancy might be due to the different anti-tumor mechanisms induced by Chidamide and 5-Fu, as 5-Fu is more efficient in inducing DNA damage and Chidamide appears to be more efficient in regulating Caspase-3, PARP, p53, p-p53-S15, p21 and CDK4. Besides, we could not exclude the possibility that 5-Fu may exert antitumor activities through other pathways.

In our preliminary animal study, we found that $5-\mathrm{Fu}$, but not chidamide, could induce diarrhea in animals after therapy. In this study, one mouse in chidamide group had mild diarrhea but no significant body weight loss was found. However, some animals in combined treatment group showed severe diarrhea and body weight loss. We speculate that the diarrhea might be induced by the administration of 5-Fu. In addition, phase I clinical trials showed that chidamide was generally well tolerated in patients with advanced solid tumors or lymphomas [13]. Phase II clinical trials showed that chidamide represented a novel oral benzamide class of HDAC inhibitor with significant single-agent activity and manageable toxicity in relapsed or refractory peripheral T-cell lymphoma (PTCL) [14]. These evidences indicate chidamide is relatively safe and has a promising therapeutic value for clinical use. Nevertheless, pre-clinical and clinical trials are needed to test the therapeutic effects of chidamide in other malignant solid tumors including colorectal cancers.

In summary, the present study showed that chidamide increased the anti-tumor effects of 5-Fu in nude mouse bearing human colon cancer xenografts. Moreover, treatment of tumor xenografts with chidamide resulted in down-regulation of AKT-mTOR and Raf-MEK-ERK signaling, both of which are the crucial cell survival signaling pathways. Intriguingly, we found that chidamide induced the phosphorylation of $\mathrm{H} 2 \mathrm{AX}$ and p53 while increased the levels of p21. The results of the present study imply that induction of DNA damage might be one of the mechanisms by which chidamide inhibits cancer cell proliferation.

Acknowledgements: We thank Chipscreen Biosciences (Shenzhen, China) for providing the Chidamide used in the present study. This research was supported by grants from Nanjing City Funds for the Development of Science and Technology of Medicine (No. ZKX07005). The funders had no role in study design, data collection and analysis, the decision to publish, and preparation of the manuscript.

\section{References}

[1] AHNEN DJ, WADE SW, JONES WF, SIFRI R, MENDOZA SILVEIRAS J, et al. The Increasing Incidence of young-onset colorectal cancer: a call to action. Mayo Clin Proc 2014; 89: 216-224. http://dx.doi.org/10.1016/j.mayocp.2013.09.006 
[2] SONG X, ZHAO Z, BARBER B, GREGORY C, SCHUTT D, et al. Characterizing medical care by disease phase in metastatic colorectal cancer. J Oncol Pract 2011; 7(3Suppl): 25s-30s. http://dx.doi.org/10.1200/JOP.2011.000304

[3] TEJANI MA, TER VEER A, MILNE D, OTTESEN R, BEKAIISAAB T, et al. Systemic therapy for advanced appendiceal adenocarcinoma: an analysis from the NCCN Oncology Outcomes Database for colorectal cancer. J Natl Compr Canc Netw 2014; 12: 1123-1130

[4] HANAHAN D, WEINBERG RA. Hallmarks of cancer: the next generation. Cell 2011; 144: 646-674. http://dx.doi. org/10.1016/j.cell.2011.02.013

[5] DU W, ELEMENTO O. Cancer systems biology: embracing complexity to develop better anticancer therapeutic strategies. Oncogene 2014; Sep 15. [Epub ahead of print] http://dx.doi. org/10.1038/onc.2014.291

[6] LAKSHMAIAH KC, JACOB LA, APARNA S, LOKANATHA D, SALDANHA SC. Epigenetic therapy of cancer with histone deacetylase inhibitors. J Cancer Res Ther 2014; 10: 469-478. http://dx.doi.org/10.4103/0973-1482.137937

[7] BOSE P, DAI Y, GRANT S. Histone deacetylase inhibitor (HDACI) mechanisms of action: emerging insights. Pharmaco Ther 2014; 143: 323-336. http://dx.doi.org/10.1016/j. pharmthera.2014.04.004

[8] KARCZMARSKI J, RUBEL T, PAZIEWSKA A, MIKULA $\mathrm{M}$, BUJKO $\mathrm{M}$, et al. $\mathrm{H} 3$ lysine 27 acetylation is altered in colon cancer. Clin Proteomics 2014; 11: 24. http://dx.doi. org/10.1186/1559-0275-11-24

[9] YANG D, TORRES CM, BARDHAN K, ZIMMERMAN M, MCGAHA TL, et al. Decitabine and vorinostat cooperate to sensitize colon carcinoma cells to Fas ligand-induced apoptosis in vitro and tumor suppression in vivo. J Immunol 2012; 88: 4441-4449. http://dx.doi.org/10.4049/jimmunol.1103035

[10] SLINGERLAND M, GUCHELAAR HJ, GELDERBLOM H. Histone deacetylase inhibitors: an overview of the clinical studies in solid tumors. Anticancer Drugs 2014; 25: 140-149. http://dx.doi.org/10.1097/CAD.0000000000000040

[11] IWAHASHI S, UTSUNOMIYA T, IMURA S, MORINE Y, IKEMOTO T, et al. Effects of valproic acid in combination with S-1 on advanced pancreatobiliary tract cancers: clinical study phases I/II. Anticancer Res 2014; 34: 5187-5191.

[12] DEL BUFALO D, DESIDERI M, DE LUCA T, DI MARTILE M, GABELLINI C, et al. Histone deacetylase inhibition synergistically enhances pemetrexed cytotoxicity through induction of apoptosis and autophagy in non-small cell lung cancer. Mol Cancer 2014; 13: 230. http://dx.doi.org/10.1186/1476-4598$\underline{13-230}$

[13] DONG M, NING ZQ, XING PY, XU JL, CAO HX, et al. Phase study of chidamide (CS055/HBI-8000), a new histone deacetylase inhibitor, in patients with advanced solid tumors and lymphomas. Cancer Chemother Pharmacol 2012; 69: 1413-1422. http://dx.doi.org/10.1007/s00280-012-1847-5
[14] SHI Y, DONG M, HONG X, ZHANG W, FENG J, et al. Results from a multicenter, open-label, pivotal phase II study of chidamide in relapsed or refractory peripheral T-cell lymphoma. Ann Oncol 2015; 26: 1766-1771. http://dx.doi: 10.1093/ annonc/ mdv237

[15] LIU L, CHEN B, QIN S, LI S, HE X, et al. A novel histone deacetylase inhibitor Chidamide induces apoptosis of human colon cancer cells. Biochem Biophys Res Commun 2010; 392: 190-195. http://dx.doi.org/10.1016/j.bbrc.2010.01.011

[16] YAO Y, ZHOU J, WANG L, GAO X, NING Q, et al. Increased PRAME-specific CTL killing of acute myeloid leukemia cells by either a novel histone deacetylase inhibitor chidamide alone or combined treatment with decitabine. PLoS One 2013; 8: e70522. http://dx.doi.org/10.1371/journal.pone.0070522

[17] GONG K, XIE J, YI H, LI W. CS055 (Chidamide/HBI-8000), a novel histone deacetylase inhibitor, induces G1 arrest, ROSdependent apoptosis and differentiation in human leukaemia cells. Biochem J 2012; 443: 735-746. http://dx.doi.org/10.1042/ B]20111685

[18] WANG H, GUO Y, FU M, LIANG X, ZHANG X, et al. Antitumor activity of Chidamide in hepatocellular carcinoma cell lines. Mol MED Rep 2012; 5: 1503-1508. http://dx.doi. org/10.3892/mmr.2012.858

[19] ZHAO B, HE T. Chidamide, a histone deacetylase inhibitor, functions as a tumor inhibitor by modulating the ratio of Bax/Bcl-2 and P21 in pancreatic cancer. Oncol Rep 2014; 33: 304-310. http://dx.doi.org/10.3892/or.2014.3595

[20] ZHOU Y, PAN DS, SHAN S, ZHU JZ, ZHANG K, et al. Non-toxic dose chidamide synergistically enhances platinum-induced DNA damage responses and apoptosis in Non-Small-Cell lung cancer cells. Biomed Pharmacother 2014; 68: 483-491. http://dx.doi.org/10.1016/j.biopha.2014.03.011

[21] CICCIA A, ELLEDGE SJ. The DNA damage response: making it safe to play with knives. Mol Cell 2010; 40: 179-204. http:// dx.doi.org/10.1016/j.molcel.2010.09.019

[22] KASTAN MB, BARTEK J. Cell-cycle checkpoints and cancer. Nature 2004; 432: 316-323. http://dx.doi.org/10.1038/ $\underline{\text { nature03097 }}$

[23] CIMPRICH KA, CORTEZ D. ATR: an essential regulator of genome integrity. Nat Rev Mol Cell Biol 2008; 9: 616-627. http://dx.doi.org/10.1038/nrm2450

[24] LOPES M, COTTA-RAMUSINO C, PELLICIOLI A, LIBERI G, PLEVANI P, et al. The DNA replication checkpoint response stabilizes stalled replication forks. Nature 2001; 412: 557-561. http://dx.doi.org/10.1038/35087613

[25] BUDANOV AV, KARIN M. p53 target genes sestrin 1 and sestrin 2 connect genotoxic stress and mTOR signaling. Cell 2008; 134: 451-460. http://dx.doi.org/10.1016/j.cell.2008.06.028

[26] VIJAYAKURUP V, CARMELA S, CARMELO D, CORRADO T, SRINIVAS P, et al. Phenethyl caffeate benzo[kl]xanthene lignan with DNA interacting properties induces DNA damage and apoptosis in colon cancer cells. Life Sci 2012; 91: 1336-1344. http://dx.doi.org/10.1016/j.lfs.2012.10.013 